\title{
Association between depression and sleep quality in patients with systemic lupus erythematosus: a systematic review and meta-analysis
}

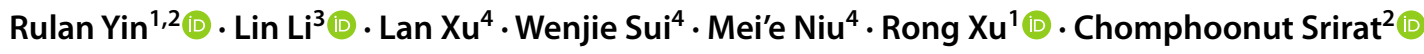

Received: 28 March 2021 / Revised: 10 May 2021 / Accepted: 15 May 2021 / Published online: 25 May 2021

(c) The Author(s) 2021, corrected publication 2021

\begin{abstract}
Background Currently, there is no consistent understanding of the relationship between depression and sleep quality in patients with systemic lupus erythematosus (SLE). This study aimed to explore the correlation between depression and sleep quality in SLE patients.

Methods Five English (PubMed, Web of Science, EMBASE, Cochrane Library, and CINAHL) databases were systematically searched from inception to January 12, 2021. Two authors independently screened publications and extracted data according to set inclusion and exclusion criteria. Statistical analyses were performed with STATA 16.0. Data were pooled using a random-effects model.

Results A total of 9 identified studies matched the inclusion criteria, reporting on 514 patients with SLE in the analysis. A moderate correlation of depression with sleep quality was found (pooled $r=0.580[0.473,0.670]$ ). Compared to good sleepers, patients with SLE and poor sleep quality had higher levels of depression (standardized mean difference $=-1.28$ $[-1.87,-0.69])$. Depression was associated with subjective sleep quality $(r=0.332[0.009,0.592])$, sleep latency $(r=0.412$ $[0.101,0.649])$, sleep disturbances $(r=0.405$ [0.094, 0.645]), daytime dysfunction $(\mathrm{r}=0.503$ [0.214, 0.711]), the four dimensions of Pittsburgh Sleep Quality Index (PSQI), while no significant correlation was found in the other three PSQI dimensions. Conclusion Depression had a moderate correlation with sleep quality in patients with SLE. Patients with poor sleep quality tended to have higher level of depression than that of good sleepers. Awareness of the correlation may help rheumatology physicians and nurses to assess and prevent depression and improve sleep quality in patients with SLE.
\end{abstract}

Keywords Depression $\cdot$ Sleep quality $\cdot$ Systemic lupus erythematosus $\cdot$ Meta-analysis

\section{Introduction}

Rulan Yin, Lin Li and Lan Xu contributed equally to this work.

Rong Xu

szronxu@sina.com

$\triangle$ Chomphoonut Srirat

chomphoonut.s@cmu.ac.th

1 Department of Rheumatology, The First Affiliated Hospital of Soochow University, 899th Pinghai Road, Suzhou 215006, China

2 Faculty of Nursing, Chiang Mai University, 110/406 Inthavaroros Road, Suthep, Muang District, Chiangmai 50200, Thailand

3 School of Nursing, Taizhou Polytechnic College, Taizhou, China

4 Department of Nursing, The First Affiliated Hospital of Soochow University, Suzhou, China
Systemic lupus erythematosus (SLE), the most common form of lupus, is a chronic, multi-organ, systemic autoimmune disease with unknown etiology and heterogeneous clinical presentation, affecting almost all tissues and organ systems, occurring predominantly in young adult women, with a female-male ratio of more than 9:1 [1,2]. It was found in a 2020 meta-analysis that sleep quality of SLE patients is worse than that of the general population [3]; 56.0-80.5\% of patients with SLE reported sleep disturbances and poor sleep quality [4]. Previous studies reported that sleep disturbances may lead to the aggravation of cardiovascular disease in patients with SLE [5]. Lack of sleep indicates a deterioration of disease activity [6]. Therefore, it is of great significance to identify the causes of poor sleep in patients with SLE so that targeted interventions can be taken 
to improve their sleep quality. However, the causes of sleep disorders have not been completely identified [7]. Meanwhile, psychological/social factors, particular depression, have been observed as the most frequent possible causes of sleep disorders in SLE [8-11]. As one of the most frequently observed neuropsychiatric disorders [12], depression affects $35.0 \%$ of patients with SLE [13]. In addition, SLE patients have more depressive symptoms than healthy women [14]. Cervilla et al. [14] reported that worse subjective quality of sleep was associated with more depressive symptoms in SLE. Wu et al. [3] also displayed that depressed patients with SLE have poorer sleep quality than that of the general population. However, Moraleda et al. [15] did not find a significant correlation with depression and sleep quality in patients with SLE. Given the importance of a clear association between depression and sleep quality in patients with SLE, it is necessary to conduct a synthesis on the empirical studies addressing this topic.

The Pittsburgh Sleep Quality Index (PSQI) [16], which consists of 19 items, is the most widely used instrument to evaluate sleep quality in clinical populations. The PSQI assesses seven dimensions: subjective sleep quality, sleep latency, sleep duration, habitual sleep efficiency, use of sleeping medication, sleep disturbances, and daytime dysfunction. Its clinemetric and clinical properties suggest utility both in psychiatric clinical practice and research activities, and has been widely applied to assess sleep quality in patients with SLE. However, despite having been measured in many studies, the degree of sleep quality in each domain has differed among different samples [3]. To the best of our knowledge, there is no comprehensive review on the relationship between depression and sleep quality as assessed by the PSQI in patients with SLE. Thus, with accumulating evidence, we performed a systematic review and meta-analysis to answer the following two questions: (i) Is there an association between depression and sleep quality in patients with SLE and (ii) Is the depression level higher in groups with poor sleep quality compared to good sleep quality.

\section{Methods}

The systematic review and meta-analysis was conducted in accordance with the recommendations of the Preferred Reporting Items for Systemic Review and Meta-Analyses (PRISMA) guidelines [17].

\section{Search strategy}

Up to January 12, 2021, five English databases were searched, including PubMed, Web of Science, EMBASE, Cochrane Library, and CINAHL. The search terms were combinations of the following: sleep (sleep problems or sleep disturbances or sleep quality or sleep efficiency or sleep latency or sleep difficulties), depression (depression or major depression disorder or depressive symptoms or mood disorder), and SLE (lupus or SLE or systemic lupus erythematosus). The reference lists of included studies were also reviewed for additional studies.

\section{Inclusion and exclusion criteria}

Original studies were included if they (1) were cross-sectional or case-control or longitudinal studies (only the data at baseline were extracted) on patients with SLE patients; (2) reported the association between depression and sleep quality with the Pearson or Spearman correlation coefficient $(r)$, or mean \pm standard deviation (SD) of depression on both good sleep quality group and poor sleep quality group; (3) used PSQI to evaluate sleep quality; (4) used a validated scale to measure depression; (5) were published in the English language.

Exclusion criteria were the following: (1) studies were meta-analyses, reviews, case reports, qualitative studies, comments, editorial letters, or conference abstracts; (2) studies did not meet the inclusion criteria; (3) studies reported unusable or duplicate data. When there were different studies in the same unit and the same sample, the most recent was selected.

\section{Data extraction and quality assessment}

According to the titles and abstracts, two authors independently decided whether to include articles by reading the abstract and further full-text examination, and independently extracted the following information from each article: first author, publication year, country, study design, aim, diagnostic criteria of SLE, source of the patient, sample size and percentage of female participants, age, disease duration, disease activity, corticosteroids use, cumulative disease damage, measurement, cut-point, prevalence and score of depression, cut-point and score of PSQI, prevalence of poor sleep quality, Pearson's $r$ or Spearman's correlation coefficient $(r)$ between depression and sleep quality, depression score both in good and poor sleep quality, and the number of patients in each group (good and poor sleep quality). Quality assessment was conducted alongside data extraction using a modified version of the Newcastle-Ottawa Scale (M-NOS) [18]. Studies were judged to be at low risk of bias ( $\geq 3$ points) or high risk of bias ( $<3$ points). Any disagreements in data extraction and quality assessment were resolved through discussion between the two reviewers or adjudication with a third reviewer. 


\section{Outcome measures}

The outcomes were depression assessed with validated assessment tools (e.g., Hospital Anxiety and Depression Scale-Depression [HADS-D], the nine-item Patient Health Questionnaire [PHQ-9], Center for Epidemiological Studies Depression scale [CES-D], Hamilton rating scale for depression [HAM-D]), and sleep quality assessed by PSQI.

\section{Statistical analysis}

Meta-analysis was performed with STATA 16. As randomeffects model was preferable and could provide wider confidence interval (CI) [19], this model was conducted to pool $r$ and continuous outcomes in this study. For correlation coefficients, Pearson correlation coefficients and Spearman correlation coefficients were all transformed into Pearson correlation coefficients. Pooled estimates of the Pearson coefficients were calculated by Fisher's exact test $r$-to- $z$ transformation [20]. All of the values were weighted with the inverse of the variance of the correlation coefficients, after which, the pooled $r$ of the overall value were transformed back for presentation. For the continuous outcomes, we used standardized mean difference (SMD) with 95\% CIs. $I^{2}$ was used to assess between-study heterogeneity, with thresholds of $25 \%$ (low heterogeneity), $50 \%$ (moderate heterogeneity), and 75\% (high heterogeneity) [21]. As the seven dimensions of PSQI indicated different aspects of sleep quality, this study separately summarized and analyzed 7 domains. Sensitivity analysis and subgroup analysis were performed to find the source of heterogeneity. Funnel plots and the Egger's test were combined to evaluate for publication bias if the number of included studies is greater than or equal to $10[22,23]$, as the power of these tests is too low to distinguish chance from real asymmetry if less than 10 studies are included [24].

\section{Results}

\section{Study selection}

After having assessed the studies by selection criteria, data from 9 studies were included, which involves 514 patients with SLE. A flow chart of the study selection process is shown in Fig. 1.

\section{Study characteristics}

Tables 1 and 2 showed the summary of the included study characteristics. Two took place in Spain [14, 15], and one occurred in each of the following countries: India [25], Canada [26], Thailand [27], Egypt [28], Iran [29],
Korea [30], and UK [31]. Two studies had large sample sizes ( $\geq 100$ cases) and the others were small sample size ( $<100$ cases). When evaluated by M-NOS, out of 5 possible points, one received 4 points, five received 3 points, and three received 2 points.

\section{Correlation between depression and sleep quality in SLE patients}

As shown in Fig. 2, eight studies [14, 15, 25-28, 30, 31] reported $r$ between depression and sleep quality, the pooled $z$ was 0.66 (95\% CI $0.51-0.81, P=0.043$; $\left.I^{2}=51.7 \%\right)$. The correlation magnitude is moderate (pooled $r=0.580$ ) and significant, which is reflected by the $95 \%$ CI $(0.473-0.670)$ that does not include the value 0 , suggesting a positive relationship. Among the seven dimensions of PSQI, depression was associated with subjective sleep quality (pooled $r=0.332,95 \%$ CI $0.009-0.592, P=0.044 ; I^{2}=0 \%$ ), sleep latency (pooled $r=0.412,95 \%$ CI $0.101-0.649, P=0.011 ; I^{2}=0 \%$ ), sleep disturbances (pooled $r=0.405,95 \%$ CI $0.094-0.645$, $P=0.012 ; I^{2}=0 \%$ ), and daytime dysfunction (pooled $r=0.503,95 \%$ CI $0.214-0.711, P=0.001 ; I^{2}=0 \%$ ), no significant correlation was found with the other three dimensions-sleep duration, habitual sleep efficiency, and use of sleeping medication (Fig. 3).

\section{Comparison of depression levels in patients with SLE and good and poor sleep quality}

There were 4 studies that compared depression between good $($ total sample size $=84)$ and poor (total sample size $=129)$ sleep quality in patients with SLE [25, 27-29]. Compared to good sleepers, patients with SLE and poor sleep quality had higher levels of depression (pooled SMD $=-1.28,95 \% \mathrm{CI}$ [-1.87, -0.69], $P<0.001 ; I^{2}=71 \%$ ) (Fig. 4).

\section{Subgroup analysis}

Subgroup analysis was conducted based on corticosteroids use, measurement of depression, diagnostic criteria of SLE, region, and quality. Table 3 indicated that corticosteroids use, measurement of depression, diagnostic criteria of SLE, and region might be the source of heterogeneity in metaanalysis of the correlation between the depression and sleep quality in SLE. As shown in Table 4, depression measurement, SLE diagnostic criteria, and study's quality may be a source of heterogeneity in meta-analysis comparing the depression level in SLE patients with good and poor sleep quality, but not including corticosteroids use and region. 


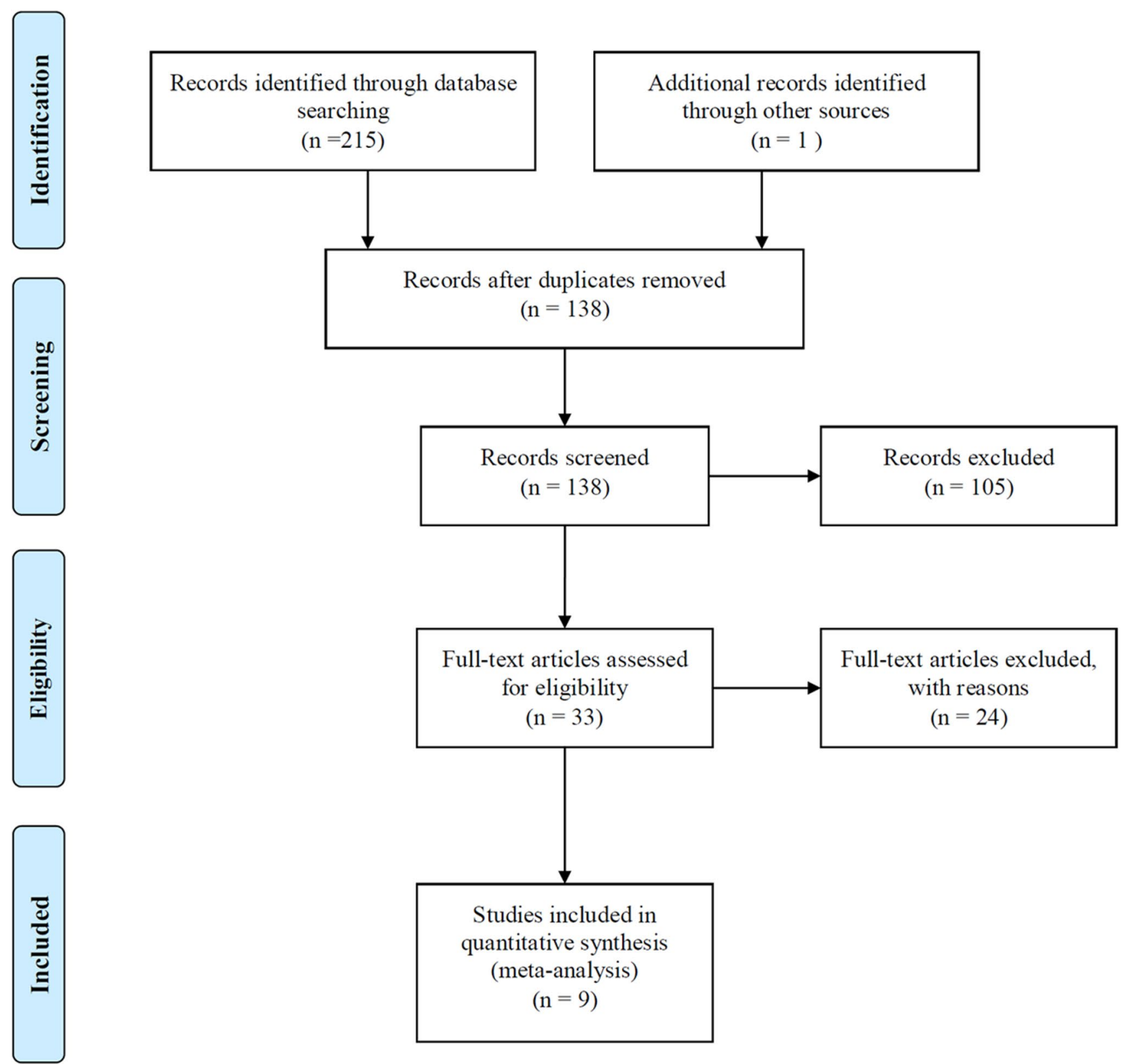

Fig. 1 Flow-chart illustrating the article search process

\section{Sensitivity analyses and publication bias}

Sensitivity analyses displayed that, for all comparisons between patients with good and poor sleep quality, as well as the pooled results of differences between depression level and sleep quality in patients with SLE, the omission of any study made no significant difference, indicating the stability of our meta-analysis (figures not shown). As the number of studies included in this meta-analysis was 9, less than 10, funnel plots and the Egger's test were not conducted to assess publication bias.

\section{Discussion}

This systematic review and meta-analysis of 9 studies involving 514 patients with SLE is the first to explore the relationship between depression and sleep quality.

In this meta-analysis, a moderate correlation of sleep quality with depression was found (pooled $r=0.580$ ). Compared to good sleepers, patients with SLE and poor sleep quality had higher levels of depression, corroborated by past studies [25, 27-29]. This study also found that there was correlation between depression and subjective sleep quality, 


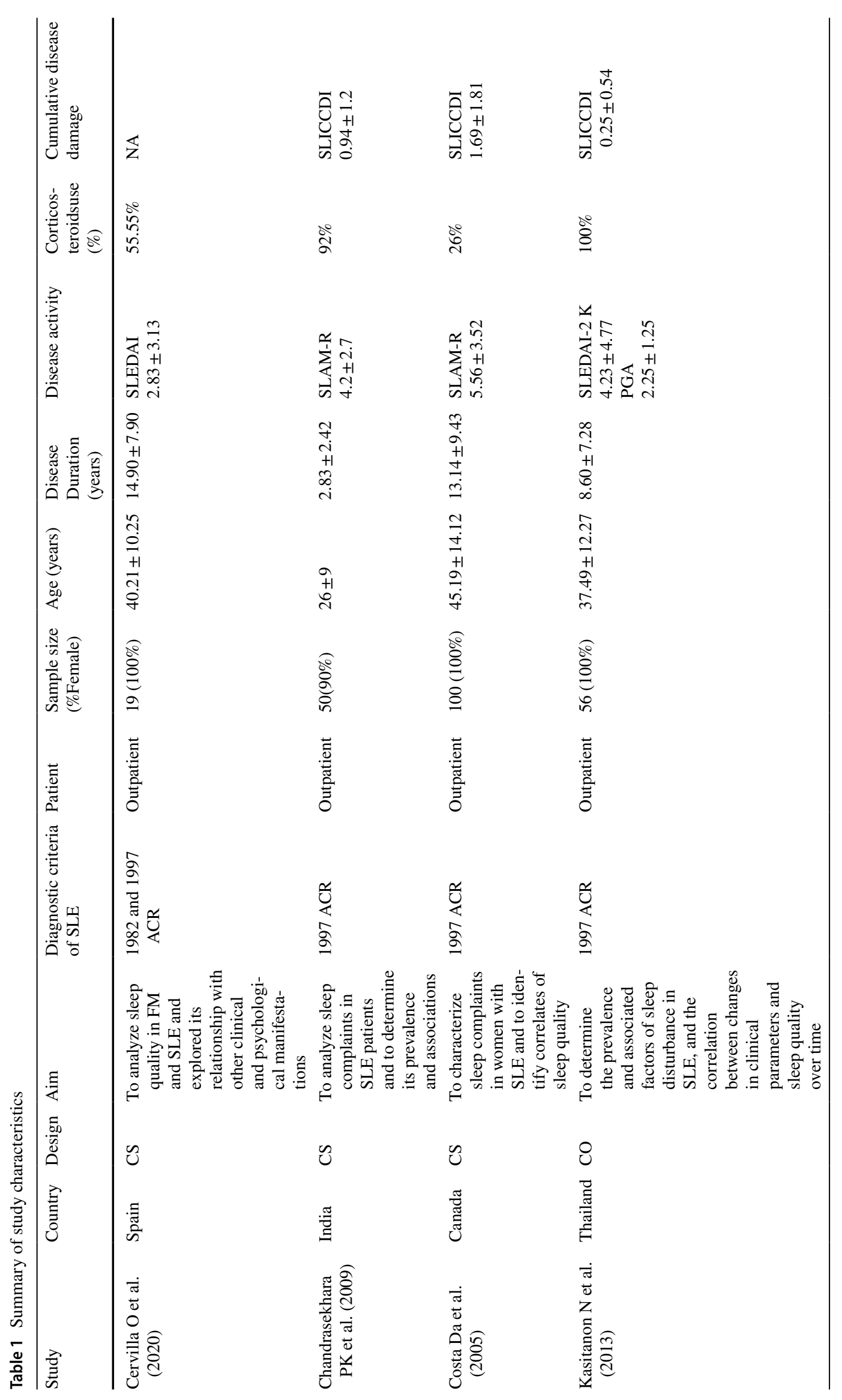




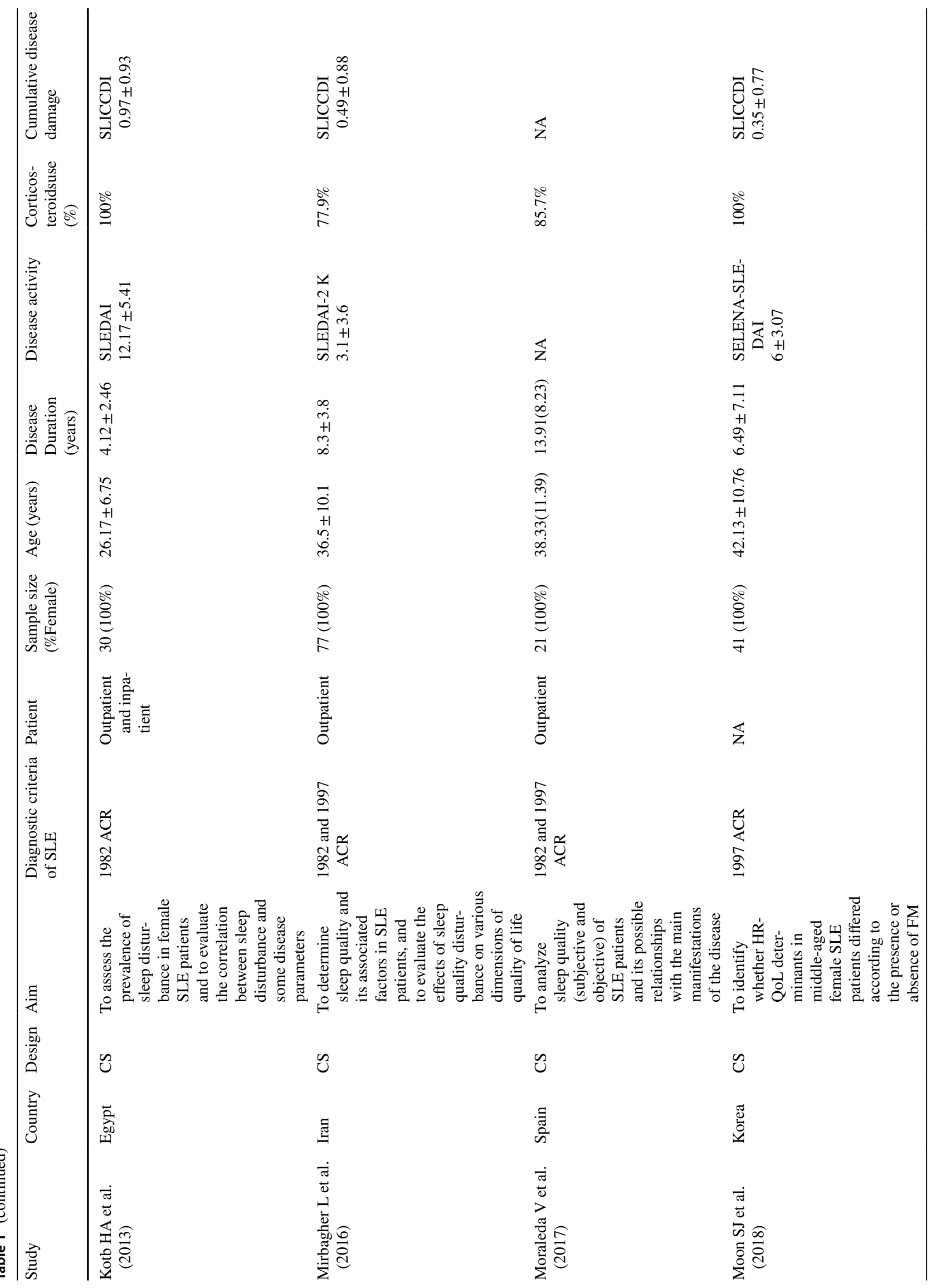



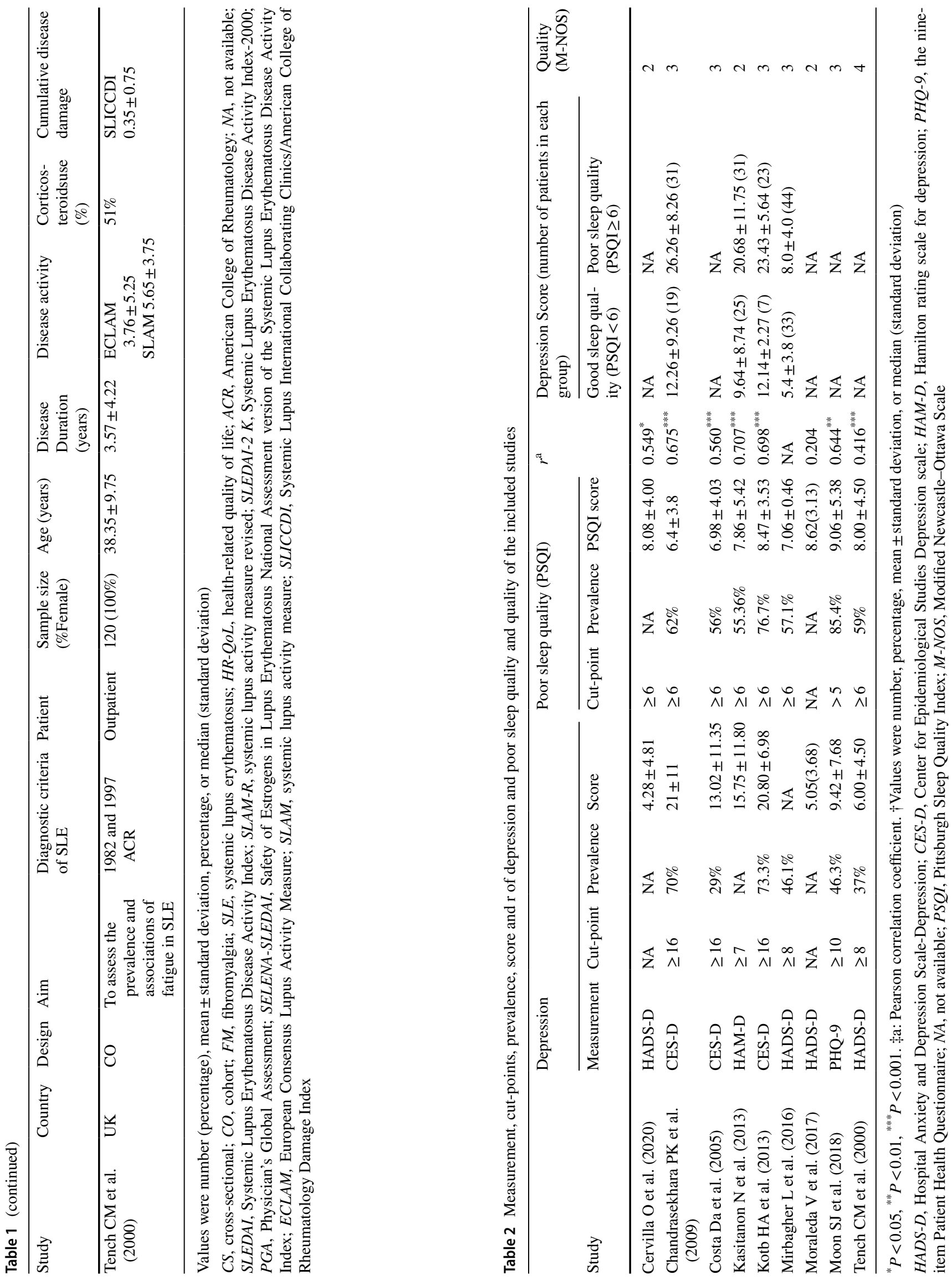
Study (Year)

Fisher z $(95 \%$ CI) Weight

Cervilla O et al (2020)

Chandrasekhara PK et al (2009)

Costa Da et al (2005)

Kasitanon $\mathrm{N}$ et al (2013)

Kotb HA et al (2013)

Moraleda V et al (2017)

Moon SJ et al (2018)

Tench CM et al (2000)

Overall, DL $\left(\mathrm{I}^{2}=51.7 \%, \mathrm{p}=0.043\right)$

NOTE: Weights are from random-effects model

$0.62(0.13,1.11)$

6.83

$0.82(0.53,1.11)$

13.31

$0.63(0.43,0.83)$

17.81

$0.88(0.61,1.15) \quad 14.09$

$0.86(0.49,1.24) \quad 9.76$

$0.21(-0.26,0.67)$

7.44

$0.76(0.45,1.08)$

11.93

$0.44(0.26,0.62)$

18.84

$0.66(0.51,0.81)$

100.00

Fig. 2 Meta-analysis of the correlation between depression and sleep quality $(N=8)$

sleep latency, sleep disturbances, and daytime dysfunction, four dimensions of the PSQI. There were no significant correlations with the other three dimensions-sleep duration, habitual sleep efficiency, and use of sleeping medication. The underlying mechanisms of sleep disorders in patients with SLE are yet unclear [4]. Previous studies have focused on possible neural mechanisms and found that sleep deprivation influences brain systems that are involved in emotion, such as the amygdala [32], and the anterior cingulate cortex are thought to be a region where genes that control circadian rhythms are dysregulated in depression [33] and where neurons increase their activity during sleep and disengagement from tasks [34]. Cheng et al. [35] found that both poor sleep quality and depressive problems are significantly positively correlated with functional connectivities involving the lateral orbitofrontal cortex, the dorsolateral prefrontal cortex, the cingulate cortex, and the precuneus; and the functional connectivity links between the brain areas identified played a significant role in the association of depressive problems with poor sleep quality, while the associations of sleep quality with depressive problems mediated by these links were less significant. These findings provides a neural basis for understanding how depression is associated with poor sleep quality, and this in turn has implications for treatment because of the brain areas identified.

Subgroup analysis demonstrated a positive correlation between depression and sleep quality in SLE patients with regard to corticosteroids use, measurement of depression, diagnostic criteria of SLE, and region; and the four variables might be the source of heterogeneity. Compared with less than $100 \%$ use of corticosteroids, SLE patients with $100 \%$ corticosteroids use had a stronger relationship between depression and sleep quality. Previous studies reported that corticosteroids can contribute to sleep difficulties and some psychiatric symptoms [26, 28]. It could be the side effects of corticosteroids, such as night sweating which affects or aggravates SLE patients' sleep disorders, and centripetal obesity that leads to body image concerns. The strength of the correlation between depression and sleep quality varied among different measurement tools of depression, with HAM-D being the high relationship and HADS-D being the low correlation, which was consistent 


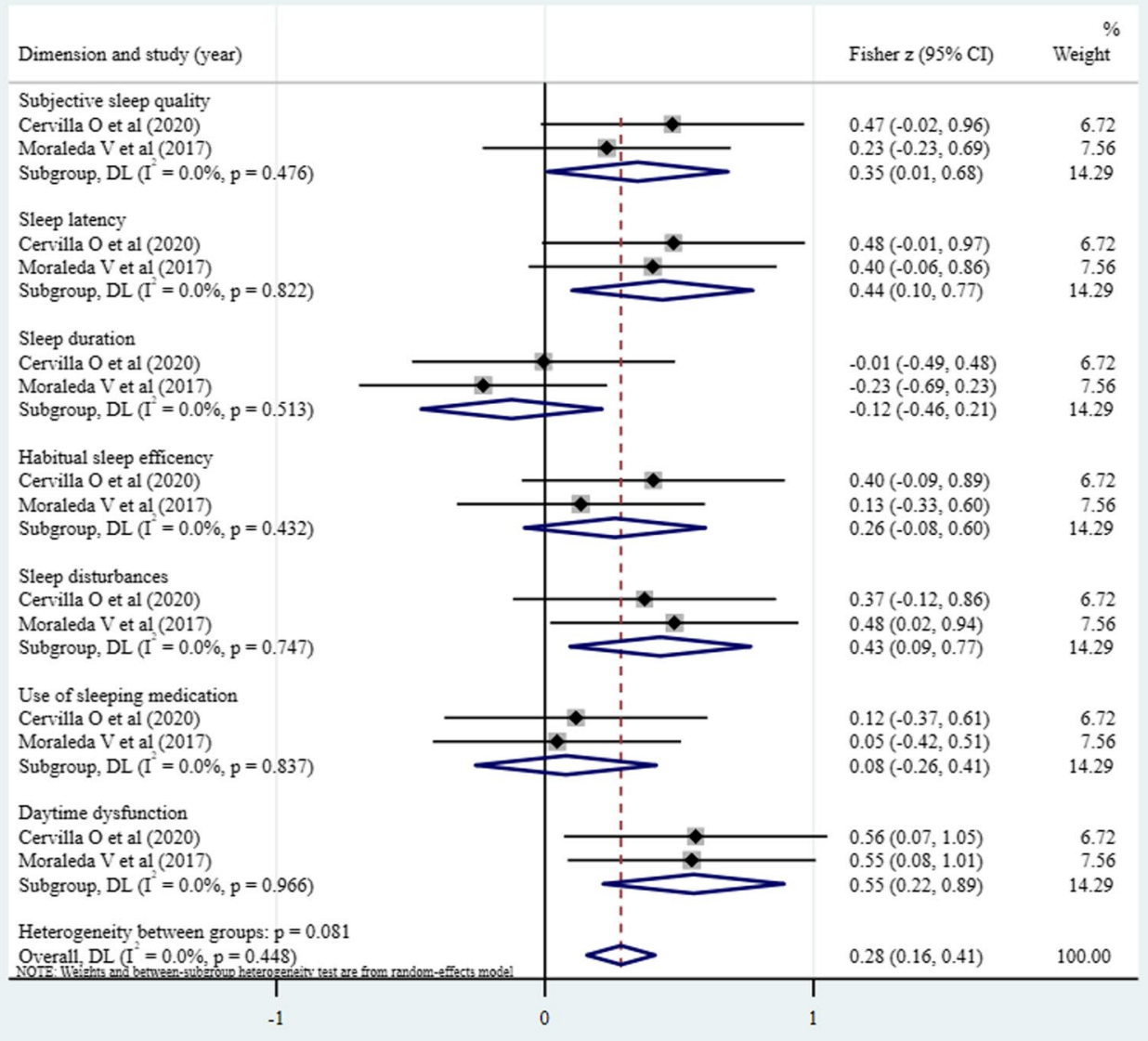

Fig. 3 Meta-analysis of the correlation between depression and 7 dimensions of PSQI $(N=2)$

with the explanation of Mirbagher et al. [29] that using different psychological measurement tools may contribute to the differences between the results. To date, subjective measurement of depression in patients with SLE has not been unified. We recommend that future investigators conduct rigorous randomized controlled trials in SLE patients to unify depression assessment tool to make the results more comparable across studies. An interesting finding was that the correlation between depression and sleep quality was strongest when SLE patients met the diagnostic criteria of 1982 ACR, followed by 1997 ACR, and weakest when patients with SLE matched both 1982 and 1997 ACR. As is well known, 1997 ACR revised the criterion number 10 of 1982 ACR- “Immunologic disorder" by deleting item 10 (a) and changing item 10(d) [36], and the sensitivity of revised "Immunologic disorder" remained the same, but the specificity decreased [37]. That is, the rate of misdiagnosis of immune abnormalities in 1982 ACR was low, which may lead to patients with immune abnormalities getting closer to a diagnosis of SLE, increasing their psychological burden and affecting their sleep quality. As the diagnostic criteria of SLE will be updated in real time with the progress of detection technology and other reasons, we suggest that researchers adopt the latest diagnostic criteria. There is another discovery that depression had a highest relationship with sleep quality in SLE patients in Africa, and lowest correlation in Europe. It may be because of the poor economic conditions and backward medical conditions in Africa that SLE patients may not get timely and effective treatment, leading to the deterioration of the disease, increased psychological burden, and restless sleep at night. In contrast, Europe has developed economy, advanced medical level and high medical insurance coverage, so the treatment of SLE patients can be carried out in an orderly manner. This review showed that literature's quality did not affect the association between depression and sleep quality in patients with SLE and might not be the source of heterogeneity of the pooled correlation coefficient. However, given that the higher the quality of the article, the more reliable the conclusions, we still hope researchers in future studies could write the manuscript according to the highest quality standards as possible. 


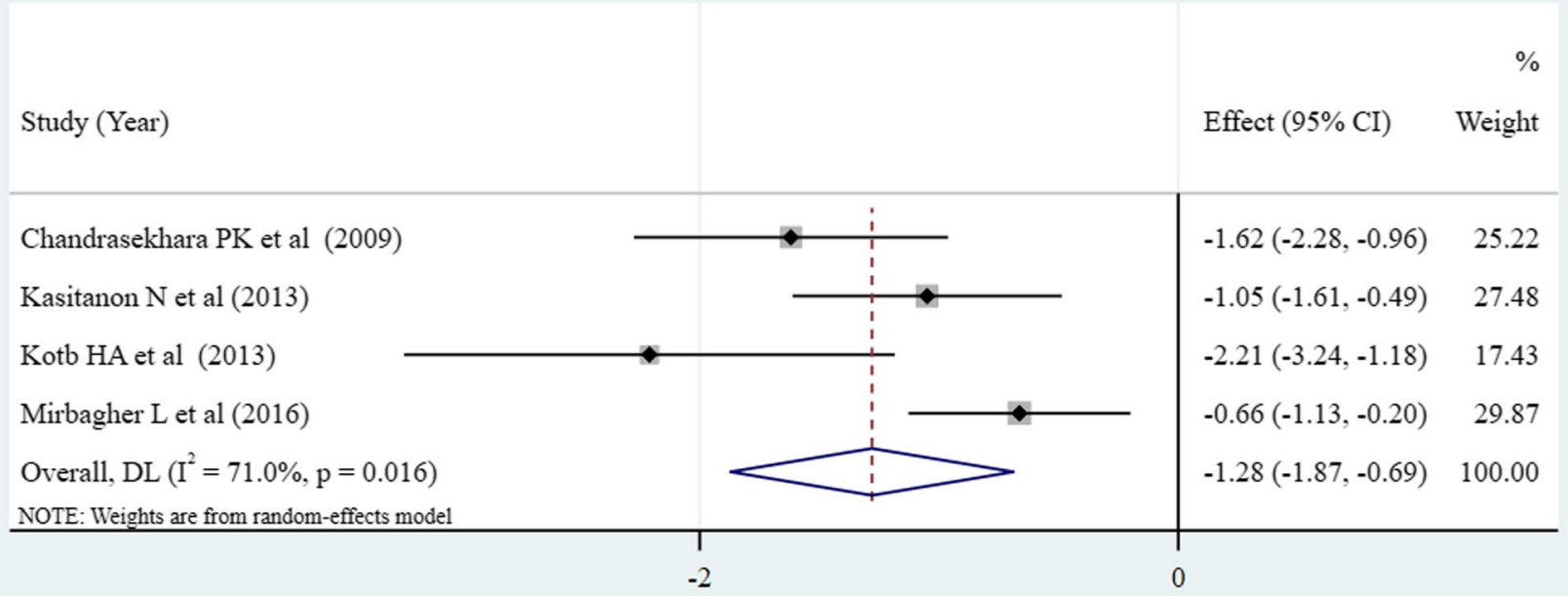

Fig. 4 Meta-analysis comparing the depression levels in patients with SLE and good sleep quality and patients with SLE and poor sleep quality $(N=4)$

Since the relationship between depression and sleep quality in SLE patients is considered to be clearer in this meta-analysis, the development of strategies to improve sleep quality in patients with SLE underscores the continuing importance of mental health assessments. Previous meta-analysis has reported the safety of therapeutic exercise programs, and exercise do not adversely affect disease activity, accompany with an interesting finding that exercise-based interventions positively influence depression [38]. Therefore, SLE patients may be able to decrease their depression levels through exercise, and thus improve their sleep quality. Lately, sleep hygiene education has shown its own potential as an intervention strategy to cover the growing public health concerns of an increasing general population with sleep complaints [39]. Nevertheless, a lack of repeated studies on the use and relevance of sleep hygiene ingredients is still existed.

However, there are several limitations in this review. First, the data were derived from studies using different designs involving different patient groups (e.g., from different countries), which may lead to heterogeneity between studies. However, we conducted subgroup analysis and sensitivity analysis to find the source of heterogeneity, and we did find some possible sources. Second, only the studies that explored sleep quality by PSQI were included, as PSQI being the most commonly used to assess SLE patient's sleep quality. Third, the assessment tools of sleep quality and depression included in this review were based on subjective feedback of the patients, which may not be objective enough. Fourth, the included researches in this meta-analysis were mainly cross-sectional designs, the number of included articles and total sample size were relatively small. Therefore, more high-quality prospective studies with large sample sizes are needed.

\section{Conclusion}

This meta-analysis suggests that depression has a moderate correlation with sleep quality in patients with SLE. Patients with poor sleep quality tended to have higher levels of 
Table 3 Subgroup analysis of the pooled results of the correlation between depression and sleep quality in SLE patients
Table 4 Subgroup analysis of the pooled results in depression levels between SLE patients with good and poor sleep quality

\begin{tabular}{|c|c|c|c|c|c|c|}
\hline \multirow[t]{2}{*}{ Variables } & \multirow{2}{*}{$\begin{array}{l}\text { No. of } \\
\text { studies }\end{array}$} & \multirow[t]{2}{*}{ No. of patients } & \multirow[t]{2}{*}{ Fisher $z(95 \% \mathrm{CI})$} & \multirow[t]{2}{*}{$P$ value } & \multicolumn{2}{|c|}{ Heterogeneity } \\
\hline & & & & & $I^{2}(\%)$ & $P$ value \\
\hline \multicolumn{7}{|l|}{ Corticosteroids use } \\
\hline$<100 \%$ & 5 & 310 & $0.57(0.39,0.74)$ & $<0.001$ & 47.3 & 0.108 \\
\hline $100 \%$ & 3 & 127 & $0.84(0.66,1.02)$ & $<0.001$ & 0 & 0.853 \\
\hline \multicolumn{7}{|c|}{ Measurement of depression } \\
\hline HADS-D & 3 & 160 & $0.43(0.27,0.59)$ & $<0.001$ & 0 & 0.479 \\
\hline CES-D & 3 & 180 & $0.72(0.57,0.87)$ & $<0.001$ & 0 & 0.415 \\
\hline HAM-D & 1 & 56 & $0.88(0.61,1.15)$ & $<0.001$ & 0 & - \\
\hline PHQ-9 & 1 & 41 & $0.76(0.45,1.08)$ & $<0.001$ & 0 & - \\
\hline \multicolumn{7}{|l|}{ Diagnostic criteria of SLE } \\
\hline 1982 and 1997 ACR & 3 & 160 & $0.43(0.27,0.59)$ & $<0.001$ & 0 & 0.479 \\
\hline 1997 ACR & 4 & 247 & $0.75(0.62,0.88)$ & $<0.001$ & 0 & 0.479 \\
\hline 1982 ACR & 1 & 30 & $0.86(0.49,1.24)$ & $<0.001$ & 0 & - \\
\hline \multicolumn{7}{|l|}{ Region } \\
\hline Europe & 3 & 160 & $0.43(0.27,0.59)$ & $<0.001$ & 0 & 0.479 \\
\hline Asia & 3 & 147 & $0.83(0.66,1.00)$ & $<0.001$ & 0 & 0.859 \\
\hline North America & 1 & 100 & $0.63(0.43,0.83)$ & $<0.001$ & 0 & - \\
\hline Africa & 1 & 30 & $0.86(0.49,1.24)$ & $<0.001$ & 0 & - \\
\hline \multicolumn{7}{|l|}{ Quality } \\
\hline High risk & 4 & 236 & $0.67(0.43,0.90)$ & $<0.001$ & 62.9 & 0.044 \\
\hline Low risk & 4 & 201 & $0.66(0.44,0.89)$ & $<0.001$ & 50.5 & 0.109 \\
\hline
\end{tabular}

$H A D S-D$, Hospital Anxiety and Depression Scale-Depression; $C E S$ - $D$, Center for Epidemiological Studies Depression Scale; $H A M-D$, Hamilton Rating Scale for Depression; $P H Q-9$, the nine-item Patient Health Questionnaire; $A C R$, American College of Rheumatology; $C I$, confidence interval

\begin{tabular}{|c|c|c|c|c|c|}
\hline \multirow[t]{2}{*}{ Variables } & \multirow{2}{*}{$\begin{array}{l}\text { No. of } \\
\text { studies }\end{array}$} & \multirow[t]{2}{*}{ Pooled SMD (95\% CI) } & \multirow[t]{2}{*}{$P$ value } & \multicolumn{2}{|c|}{ Heterogeneity } \\
\hline & & & & $I^{2}(\%)$ & $P$ value \\
\hline \multicolumn{6}{|l|}{ Corticosteroids use } \\
\hline$<100 \%$ & 2 & $-0.11(-2.05,-0.18)$ & 0.020 & 81.6 & 0.020 \\
\hline $100 \%$ & 2 & $-1.55(-2.67,-0.42)$ & 0.007 & 73.6 & 0.052 \\
\hline \multicolumn{6}{|c|}{ Measurement of depression } \\
\hline CES-D & 2 & $-1.79-2.34,-1.24)$ & $<0.001$ & 0 & 0.341 \\
\hline HAM-D & 1 & $-1.05(-1.61,-0.49)$ & $<0.001$ & 0 & - \\
\hline HADS-D & 1 & $-0.66(-1.13,-0.20)$ & 0.005 & 0 & - \\
\hline \multicolumn{6}{|l|}{ Diagnostic criteria of SLE } \\
\hline 1982 and 1997 ACR & 1 & $-0.66(-1.13,-0.20)$ & 0.005 & 0 & - \\
\hline 1997 ACR & 2 & $-1.31(-1.86,-0.75)$ & $<0.001$ & 39.9 & 0.197 \\
\hline $1982 \mathrm{ACR}$ & 1 & $-2.21(-3.24,-1.18)$ & $<0.001$ & 0 & - \\
\hline \multicolumn{6}{|l|}{ Region } \\
\hline Asia & 3 & $-1.07(-1.60,-0.54)$ & $<0.001$ & 63.4 & 0.065 \\
\hline Africa & 1 & $-2.21(-3.24,-1.18)$ & $<0.001$ & 0 & - \\
\hline \multicolumn{6}{|l|}{ Quality } \\
\hline High risk & 2 & $-0.82(-1.19,-0.45)$ & $<0.001$ & 6.9 & 0.300 \\
\hline Low risk & 2 & $-1.79(-2.34,-1.24)$ & $<0.001$ & 0 & 0.341 \\
\hline
\end{tabular}

$C E S-D$, Center for Epidemiological Studies Depression Scale; HAM-D, Hamilton Rating Scale for Depression; HADS-D, Hospital Anxiety and Depression Scale-Depression; $A C R$, American College of Rheumatology; SMD, standardized mean difference; $C I$, confidence interval 
depression than those of good sleepers. Awareness of this correlation may prompt rheumatology physicians and nurses to assess and treat depression and improve patients' sleep quality.

Acknowledgements We would like to thank Lifen Mao, Haifang Wang, and Mingjing Fang for their great assistance in data extraction and analysis; thank Xiaoqing Shi and Mingjun Wang for their suggestions in the process of revising the manuscript.

Authors' contributions RY and LL searched and checked the databases according to the inclusion and exclusion criteria, extracted the data and assessed their quality. RY analyzed the data and wrote the draft of the paper. LL, LX, WS, MN, and RX gave advice on meta-analysis methodology and revised the paper. All authors contributed to reviewing or revising the paper. RX and CS are the guarantors of this work and had full access to all the data in the study and take responsibility for its integrity and the accuracy of the data analysis. All authors read and approved the final manuscript.

Funding This study was supported by Science, Education and Health, Youth Science and Technology Project of Suzhou City (grant nos KJXW2018007); Project of Taizhou Polytechnic College (grant nos TZYKY-19-20, TZYKY-19-12); Science and Technology Development Plan Project of Suzhou City (grant nos SYS2019043 and SYS2018035).

\section{Declarations}

Conflict of interest The authors declare that they have no conflict of interest.

Open Access This article is licensed under a Creative Commons Attribution 4.0 International License, which permits use, sharing, adaptation, distribution and reproduction in any medium or format, as long as you give appropriate credit to the original author(s) and the source, provide a link to the Creative Commons licence, and indicate if changes were made. The images or other third party material in this article are included in the article's Creative Commons licence, unless indicated otherwise in a credit line to the material. If material is not included in the article's Creative Commons licence and your intended use is not permitted by statutory regulation or exceeds the permitted use, you will need to obtain permission directly from the copyright holder. To view a copy of this licence, visit http://creativecommons.org/licenses/by/4.0/.

\section{References}

1. Golder V, Hoi A (2017) Systemic lupus erythematosus: an update. Med J Aust 206:215-220

2. Nusbaum JS, Mirza I, Shum J et al (2020) Sex differences in systemic lupus erythematosus. Mayo Clin Proc 95:384-394

3. Wu L, Shi P, Tao S, Tao J, Wu G (2020) Decreased sleep quality in patients with systemic lupus erythematosus: a meta-analysis. Clin Rheumatol

4. Palagini L, Tani C, Mauri M et al (2014) Sleep disorders and systemic lupus erythematosus. Lupus 23:115-123

5. Davies RJ, Lomer MC, Yeo SI, Avloniti K, Sangle SR, D’Cruz DP (2012) Weight loss and improvements in fatigue in systemic lupus erythematosus: a controlled trial of a low glycaemic index diet versus a calorie restricted diet in patients treated with corticosteroids. Lupus 21:649-655
6. Chu P (2018) Poor sleep quality predicts worsening SLE disease activity. Paper presented at the 2018 ACR/ARHP Annual Meeting

7. Li T, Cui C, Li Y, Wang L (2020) The impacts of resilience on the association between illness uncertainty and sleep quality among Chinese women with systemic lupus erythematosus. Clin Rheumatol 39:1609-1616

8. Ahn GE, Ramsey-Goldman R (2012) Fatigue in systemic lupus erythematosus. Int J Clin Rheumatol 7:217-227

9. Vgontzas AN, Zoumakis E, Bixler EO et al (2004) Adverse effects of modest sleep restriction on sleepiness, performance, and inflammatory cytokines. J Clin Endocrinol Metab 89:2119-2126

10. Clinton JM, Davis CJ, Zielinski MR, Jewett KA, Krueger JM (2011) Biochemical regulation of sleep and sleep biomarkers. J Clin Sleep Med 7(5 Suppl):S38-S42

11. Krueger JM (2008) The role of cytokines in sleep regulation. Curr Pharm Des 14:3408-3416

12. Saito T, Tamura M, Chiba Y et al (2017) Regional cerebral glucose metabolism in systemic lupus erythematosus patients with major depressive disorder. J Neurol Sci 379:127-130

13. Moustafa AT, Moazzami M, Engel L et al (2020) Prevalence and metric of depression and anxiety in systemic lupus erythematosus: a systematic review and meta-analysis. Semin Arthritis Rheu 50:84-94

14. Cervilla O, Miro E, Pilar Martinez M, Isabel Sanchez A, Mario Sabio J, Prados G (2020) Sleep quality and clinical and psychological manifestations in women with mild systemic lupus erythematosus activity compared to women with fibromyalgia: a preliminary study. Mod Rheumatol 30:1016-1024

15. Moraleda V, Prados G, Martínez MP, Sánchez AI, Sabio JM, Miró E (2017) Sleep quality, clinical and psychological manifestations in women with systemic lupus erythematosus. Int $\mathbf{J}$ Rheum Dis 20:1541-1550

16. Buysse DJ, Reynolds CF, Monk TH, Berman SR, Kupfer DJ (1989) The Pittsburgh sleep quality index: a new instrument for psychiatric practice and research. Psychiat Res 28:193-213

17. Moher D, Liberati A, Tetzlaff J, Altman DG (2009) Preferred reporting items for systematic reviews and meta-analyses: the PRISMA statement. J Clin Epidemiol 62:1006-1012

18. Stang A (2010) Critical evaluation of the Newcastle-Ottawa scale for the assessment of the quality of nonrandomized studies in meta-analyses. Eur J Epidemiol 25:603-605

19. Borenstein M, Hedges LV, Higgins JP, Rothstein HR (2010) A basic introduction to fixed-effect and random-effects models for meta-analysis. Res Synth Methods 1:97-111

20. HC Shadish WR (1884) Combining estimates of effect size. In: Cooper H, Hedges LV, editors. The Handbook of Research Synthesis. Russell Sage Foundation, New York, p 265-6

21. Higgins JP, Thompson SG, Deeks JJ, Altman DG (2003) Measuring inconsistency in meta-analyses. BMJ 327:557-560

22. Egger M, Smith GD, Schneider M, Minder C (1997) Bias in meta-analysis detected by a simple, graphical test. BMJ 315:629-634

23. Sterne JAC, Egger M (2001) Funnel plots for detecting bias in meta-analysis: guidelines on choice of axis. J Clin Epidemiol 54:1046-1055

24. Abhyankar A, Ham M, Moss AC (2013) Meta-analysis: the impact of disease activity at conception on disease activity during pregnancy in patients with inflammatory bowel disease. Aliment Pharm Ther 38:460-466

25. Chandrasekhara PK, Jayachandran NV, Rajasekhar L, Thomas J, Narsimulu G (2009) The prevalence and associations of sleep disturbances in patients with systemic lupus erythematosus. Mod Rheumatol 19:407-415

26. Da CD, Bernatsky S, Dritsa M et al (2005) Determinants of sleep quality in women with systemic lupus erythematosus. Arthritis Rheum 53:272-278 
27. Kasitanon N, Achsavalertsak U, Maneeton B et al (2013) Associated factors and psychotherapy on sleep disturbances in systemic lupus erythematosus. Lupus 22:1353-1360

28. Kotb HA, Rady HM, Ghanim DH (2013) Sleep disturbance in female patients with systemic lupus erythematosus and its relation to disease parameters. Egyptian Rheumatologist 35:127-132

29. Mirbagher L, Gholamrezaei A, Hosseini N, Sayed BZ (2016) Sleep quality in women with systemic lupus erythematosus: contributing factors and effects on health-related quality of life. Int $\mathbf{J}$ Rheum Dis 19:305-311

30. Moon SJ, Kang KY, Kwok SK et al (2018) Differences in quality of life determinants according to the presence of fibromyalgia in middle-aged female patients with systemic lupus erythematosus: a multicenter, cross-sectional, single-ethnicity cohort. Int J Rheum Dis 21:1173-1184

31. Tench CM, Mccurdie I, White PD, D'Cruz DP (2000) The prevalence and associations of fatigue in systemic lupus erythematosus. Rheumatology (Oxford) 39:1249-1254

32. Goldstein AN, Walker MP (2014) The role of sleep in emotional brain function. Annu Rev Clin Psychol 10:679-708

33. Bunney BG, Li JZ, Walsh DM et al (2015) Circadian dysregulation of clock genes: clues to rapid treatments in major depressive disorder. Mol Psychiatry 20:48-55
34. Gabbott PL, Rolls ET (2013) Increased neuronal firing in resting and sleep in areas of the macaque medial prefrontal cortex. Eur J Neurosci 37:1737-1746

35. Cheng W, Rolls ET, Ruan H, Feng J (2018) Functional connectivities in the brain that mediate the association between depressive problems and sleep quality. Jama Psychiat 75:1052

36. Hochberg MC (1997) Updating the American College of Rheumatology revised criteria for the classification of systemic lupus erythematosus. Arthritis Rheum 40:1725

37. Zheng Y (2013) Comparison of ACR classification criteria for Systemic Lupus Erythematosus, Fujian Medical University

38 O'Dwyer T, Durcan L, Wilson F (2017) Exercise and physical activity in systemic lupus erythematosus: a systematic review with meta-analyses. Semin Arthritis Rheu 47:204-215

39. Irish LA, Kline CE, Gunn HE, Buysse DJ, Hall MH (2015) The role of sleep hygiene in promoting public health: a review of empirical evidence. Sleep Med Rev 22:23-36

Publisher's note Springer Nature remains neutral with regard to jurisdictional claims in published maps and institutional affiliations. 